
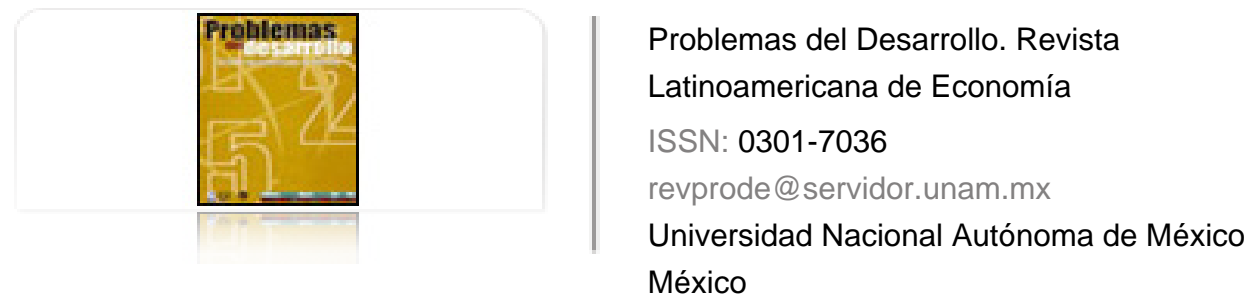

Asuad Sanén, Normand Eduardo; Quintana Romero, Luis; Ramírez Hernández, Roberto Convergencia espacial y concentración regional agrícola en México 1970-2003 Problemas del Desarrollo. Revista Latinoamericana de Economía, vol. 38, núm. 149, abril-junio, 2007, pp. 79-111

Universidad Nacional Autónoma de México

Distrito Federal, México

Disponible en: http://www.redalyc.org/articulo.oa?id=11820124005

Cómo citar el artículo

Número completo

- Más información del artículo

Página de la revista en redalyc.org

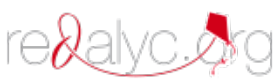

Sistema de Información Científica

Red de Revistas Científicas de América Latina, el Caribe, España y Portugal Proyecto académico sin fines de lucro, desarrollado bajo la iniciativa de acceso abierto 


\section{CONVERGENCIA ESPACIAL Y CONCENTRACIÓN REGIONAL AGRíCOLA EN MÉXICO 1970-2003}

\section{Normand Eduardo Asuad Sanén* Luis Quintana Romero** Roberto Ramírez Hernández ${ }^{* * *}$}

Fecha de recepción:12 de diciembre de 2006. Fecha de aceptación: 26 de abril de 2007.

\section{Resumen}

El propósito de este trabajo es analizar la relación entre convergencia tradicional y espacial del ingreso según la concentración regional de la producción agrícola de México, destacando los efectos propiciados por el cambio del modelo de desarrollo y gestión, mediante la liberalización y privatización de la economía con su incorporación al TLCAN y su comparación con el periodo 1970-2004. Se identifican y delimitan las regiones agrícolas a las que se aplica la medición econométrica de las hipótesis de convergencia tradicional, espacial y de la concentración económica. Los resultados confirman la tendencia a la disparidad del ingreso de esas regiones y el papel de la concentración económica como determinante de su comportamiento, asociado a la politica de apertura de la economía mexicana.

Palabras clave: convergencia tradicional, convergencia espacial, concentración económica espacial, regionalización agrícola, econometría espacial.

* Profesor de tiempo completo en la Facultad de Economía de la UNAM.

** Profesor de tiempo completo, adscrito al Programa de Investigación de la Facultad de Estudios Superiores, plantel Acatlán, de la UNAM.

*** Académico de tiempo completo del Instituto de Investigaciones Económicas de la UNAM.

1 Este trabajo es un avance de investigación sobre el comportamiento de las regiones agrícolas del país y tiene como base una serie de trabajos anteriores que se han desarrollado de manera individual y en grupo. La orientación teórica en la concepción de espacio se apoya en el trabajo de Asuad Sanén, "Un ensayo teórico y metodológico sobre el proceso de concentración económica espacial y su evidencia empírica en la región económica megalopolitana de 1970 a 2003 y sus antecedentes" (en prensa). Además de tomar como fundamento los trabajos aplicados siguientes: 
Abstract

The study proposes to analyze the relation between the traditional and spatial convergence of incomes and the regional concentration of farming production in Mexico, emphasizing the effects promoted by a changing model of development and management through the liberalization and privatization of the economy arising from the country's membership of NAFTA and a comparison with the period 1970-2004. The farm regions are identified and defined, applying econometric measurement based on the hypothesis of traditional and spatial convergence and economic concentration in geographical space. The results confirm a trend towards income disparity in those regions and the role of economic concentration as a determinant in their performance, associated with the Mexican economy's aperture policy.

Key words: Traditional convergence, spatial convergence, spatial economic concentration, farm regionalization, spatial econometrics.

\section{Résumé}

Le but de ce travail est d'analyser la relation entre convergence traditionnelle et spatiale du revenu et la concentration régionale de la production agricole du Mexique, et de mettre en évidence, par comparaison avec la période de 1970 à 2004, les effets du changement de modèle de développement et de gestion constitué par la libéralisation et privatisation de l'économie menée dans le cadre de son incorporation au TLCAN (Traité de Libre Commerce pour l'Amérique du Nord). Il est procédé à l'identification et à la délimitation des régions agricoles, auxquelles est appliquée la mesure économétrique des hypothèses de convergence traditionnelle et spatiale et de concentration économique dans l'espace géographique. Les résultats confirment la tendance à la disparité des revenus de ces régions et le rôle déterminant que joue dans leur comportement la concentration économique liée à la politique d'ouverture de l'économie mexicaine.

Mots clés: convergence traditionnelle, convergence spatiale, concentration économique régionale, régionalisation agricole, économétrie régionale.

\section{Resumo}

O propósito deste trabalho é analisar a relação entre a convergência tradicional e espacial do ingresso e a concentração regional da produção agrícola do México, destacando os efeitos propiciados pela mudança do modelo de desenvolvimento e gestão, através da liberalização e privatização da economia com a sua incorporação a NAFTA e sua comparação com o período de 1970 a 2004. Identificam-se e delimitamse as regiões agrícolas, às quais se aplica a medição econométrica das hipóteses de convergência tradicional, espacial e da concentração econômica no espaço geográfico. Os resultados confirmam a tendência à disparidade do ingresso dessas regiões e o papel da concentração econômica como determinante do seu comportamento, associado à política de abertura da economia mexicana.

Palavras-chave: Convergência tradicional, convergência espacial, concentração econômica espacial, regionalização agrícola, econometría espacial. 


\section{Introducción}

raíz del cambio hacia el actual modelo de desarrollo - basado en las expor-
taciones y en el ajuste estructural—, la economía mexicana trajo consigo la
política económica de liberalización y privatización, lo que ha dado lugar a la estabilización relativa de los principales agregados macroeconómicos. Numerosos estudios ${ }^{2}$ han señalado logros en el crecimiento de las exportaciones, inversión, empleo e incluso en el comportamiento del sector agrícola como efecto del Tratado de Libre Comercio de América del Norte (TLCAN).

El acuerdo económico firmado con Estados Unidos y Canadá en 1993 fue el instrumento con el cual la política económica y la del sector agrícola se orientó hacia el exterior. Su comportamiento, a pesar de la competencia de la agricultura estadounidense y canadiense, ha tenido un dinámico crecimiento en algunos de sus productos. $^{3}$

- "Impactos de la liberalización y de la integración económica en las regiones agrícolas en México de 1988 2004", de Normand Asuad Sanén, Luis Quintana y Roberto Ramírez Hernández (en elaboración).

- "Convergencia espacial en el crecimiento económico de las entidades federativas de México, 1940 2001”, elaborado por Normand Eduardo Asuad Sanén de la Facultad de Economía de la UNAM y Luis Quintana Romero de la FES ACATLÁN (en prensa).

- "Desarrollo y políticas regionales en México: retos y perspectivas 2006 2020", elaborado por Normand Asuad, Luis Quintana y Roberto Ramírez Hernández, (en prensa).

2 Las exportaciones mexicanas de 1993 2000 crecieron 238\% mientras que la inversión se caracterizó por una tasa de crecimiento medio anual de $12.5 \%$ de $1996-2000$, con un monto de 81 billones de dólares. Por su parte el empleo total creció $38 \%$ en el periodo, $4 \%$ anual, creándose 2.7 millones de empleos en el país, la mayor parte en el sector manufacturero con salarios $40 \%$ mayores que los que pagaba la industria nacional; además de haber propiciado un flujo creciente de inversión extranjera directa. Véase: USTR (2001). "NAFTA at Seven." Seven Year report http://www.ustr.gov/regions/whemisphere/ nafta.shtml, Washington, DC; Why NAFTA Did Not Reach the South, Gerardo Esquivel, Daniel Lederman, Miguel Messmacher y Renata Villoro, junio 12, 2002; Changes in the Patterns of External Financing in México Since the Approval of NAFTA, Alfredo Cuevas, Miguel Messmacher y Alejandro Werner, Banco de México, julio de 2002 .

3 La producción de frutas, granos, maíz y los cultivos de exportación crecieron a tasas superiores en el periodo de 1995 2004 con respecto a 1983 1994. Las frutas crecieron a una tasa 
Por otra parte, investigaciones recientes señalan que está ocurriendo una reestructuración de la agricultura mexicana, caracterizada por una elevada concentración del crecimiento en las regiones norte y occidente de la agricultura comercial del país. Se destaca también el incremento ocurrido en las desigualdades entre diferentes tipos de consumidores, urbanos y rurales, entre el norte y sur del país. ${ }^{4}$ Esto último a pesar de que la política pública ha canalizado importantes montos de gasto público y transferencias a la actividad, mediante subsidios agrícolas y soportes como ASERCA para el mercadeo, PROCAMPO y Alianza para la Producción. Además de poner en marcha programas sociales para la atención de grupos específicos mediante el programa Oportunidades y la atención a áreas remotas por medio de la descentralización del ramo presupuestal 33 y de programas ambientales. Políticas que para 2003 se estima dieron lugar a transferencias de aproximadamente 10 mil millones de pesos. ${ }^{5}$

De aquí que el propósito de esta investigación sea analizar los efectos del TLCAN en el comportamiento de las disparidades del ingreso agrícola regional, con el enfoque de convergencia espacial, en comparación con los resultados de la convergencia tradicional, que incluye como variable explicativa la concentración económica espacial. La importancia teórica y metodológica de este análisis radica en que hasta la fecha en el país no se ha realizado un estudio de este tipo, a pesar de que existen diversas aplicaciones sobre la convergencia espacial en varias naciones europeas, tal y como aquí se presenta en la revisión de la literatura.

La pregunta de investigación que orienta este trabajo corresponde a si las modificaciones de la política gubernamental, a partir de la apertura económica y de la firma del TLCAN, se han traducido en una disminución de las desigualdades regionales del crecimiento y del ingreso agrícola —en el periodo 1970-2004—y si dichas repercusiones se asocian a comportamientos sectoriales o espaciales o a ambos.

media promedio anual de $1.6 \%$, los granos a $2.2 \%$, superándolos el maíz con $2.5 \%$ y los productos de exportación a $1.3 \%$, mientras que en el periodo anterior estos productos crecieron a tasas menores: $1.0 \%, 1.1 \%, 1.1 \%, 2.0 \%$ y $0.2 \%$, respectivamente. No obstante, las oleaginosas y las hortalizas presentaron tasas negativas. Véase Nicita A., 2004, Who Benefited from Trade Liberalization in Mexico? Measuring the Effects on Household Welfare. World Bank Policy Research Paper 3676, The World Bank, Washington, DC, 55 p.

4 Losch B., Léonard E., "L'insertion de l'agriculture mexicaine dans le marché nord américain: changements structurels, mutations de l'action publique et recompositions de l'économie rurale et régionale". Colloque RINOS CEIM UQAM, Intégrations régionales et stratégies de développement, Montréal, 1 3 junio de 2005, 27 p.

5 Antonio Yúnez Naude y Fernando Barceinas Paredes (2002). 
En consecuencia, se plantean como pregunta de investigación: ¿cuáles han sido las tendencias de la convergencia en el ingreso per capita de las regiones agrícolas del país a partir de la liberalización a mediados de los años ochenta de la economía mexicana?

La hipótesis de trabajo que se propone comprobar es la siguiente: el ingreso de las regiones agrícolas del país ha tendido a mayor divergencia entre las regiones de ingresos altos y bajos, en gran medida propiciado por la concentración económica espacial de la producción, lo cual es indicativo de la incapacidad de la política gubernamental de apertura de la economía y del TLCAN, para el desarrollo del sector agrícola y sus regiones.

\section{Revisión de la literatura}

La revisión de la literatura sobre el análisis de convergencia tradicional se realiza a partir de dos vertientes críticas: la primera establece que el crecimiento económico e ingreso entre regiones ricas y pobres se caracteriza, principalmente, por su tendencia divergente, en particular mediante la formación de núcleos de convergencia de regiones ricas y pobres. Es decir, las primeras se acercan entre sí y permanecen e incluso aumentan las desigualdades en relación con las más pobres. ${ }^{6} \mathrm{El}$ segundo enfoque crítico de la convergencia tradicional corresponde a la no consideración del espacio y de la concentración económica como variables significativas en el análisis de la hipótesis de convergencia, destacando el enfoque de la econometría espacial.

\section{Convergencia tradicional y concentración económica espacial}

A partir de la literatura de Barro y Sala-i Martin (1992), la hipótesis de convergencia en el crecimiento económico se basa en un modelo econométrico que relaciona el crecimiento del PIB per capita con el nivel inicial del PIB. ${ }^{7}$ De tal forma que el crecimiento de economías ricas y pobres tiende a converger hacia el estado estacionario y la velocidad de la convergencia depende de las diferencias en el stock inicial de capital y en los rendimientos de capital decreciente. Razón por la cual las econo-

6 La ecuación de convergencia se deriva a partir de una función de producción neoclásica con el supuesto de rendimientos decrecientes del capital. La formulación de la ecuación de convergencia y sus supuestos son ampliamente conocidos por la gran difusión del tema, por ello no se desarrolla aquí y se remite al lector al libro de Sala-i-Martin (2002).

$7 \quad$ La base del enfoque corresponde al modelo neoclásico unisectorial de Solow (1956) y sus extensiones propuestas por Cass (1965), Koopmans (1965) y Diamond (1965). 
mías más pobres, con poco capital, crecerán a tasas mayores, dada su mayor productividad marginal del capital. Mientras que las economías ricas, por sus mayores dotaciones de capital, lo harán a tasas menores, en el supuesto de que la tecnología, la tasa de ahorro y población sean exógenas y constantes. La relación inversa entre el ingreso inicial y su tasa de crecimiento - en el caso de que la únicas diferencias sean sus stocks iniciales de capital— se conoce como la hipótesis de convergencia. Por lo que sí varía la tecnología, el ahorro, la depreciación o la tasa de crecimiento poblacional; la hipótesis no predice un mayor crecimiento para las regiones pobres, de ahí que no se plantee la existencia de convergencia absoluta. No obstante, se propone la presencia de convergencia condicional si la tasa de crecimiento económico de una región pobre es mayor y está directamente relacionada con la distancia a la que se sitúa de su estado estacionario, de tal manera que tenderá a su propio nivel de equilibrio en el largo plazo. Es decir, el estado estacionario puede variar de una región a otra reflejando los fundamentos económicos subyacentes. ${ }^{8}$ No obstante, varios estudios al usar metodologías semejantes critican la propensión a la convergencia condicional del ingreso de las regiones y destacan la tendencia a la concentración y a la formación de núcleos de convergencia. ${ }^{9}$ De ahí que los análisis de convergencia realizados señalen la necesidad de profundizar en el tipo de convergencia que produce el crecimiento, particularmente en la formación de clubes y en el efecto e importancia de la concentración económica espacial. No obstante que se han hecho

8 En el caso del modelo neoclásico no ortodoxo, se incorporan rendimientos crecientes; Mankiw, Romer y Weil (1992) establecen una ecuación a partir del modelo de crecimiento Solow Swan, en donde además de considerar la tecnología endógena, se amplia el concepto de capital, incorporando al capital físico el humano (Lucas, 1988) y el desarrollo de innovaciones (Grossman y Helpman, 1991 y 1994). De tal forma que al considerar esos supuestos se invierte la predicción de la convergencia del crecimiento económico neoclásico, estableciendo que lo que produce el crecimiento es divergencia, dado que las regiones más ricas crecen más rápidamente que las pobres, de tal forma que la desigualdad entre ambas tiende a aumentar con el paso del tiempo. En Sala -i-Martin (2002) se pueden consultar con detalle las características de los modelos de crecimiento mencionados.

9 Veáse Canova y Marcel (1995) (Boldrin y Canova 2001) y finalmente el estudio de Canova (2004) establece que los ingresos tienden a aglomerarse alrededor de cuatro polos de atracción con diferentes dinámicas y estados estacionarios. Los estudios europeos de núcleos de convergencia corresponden a Dunford (1993) y Esteban (1994), Dewhurst y Mutis Gaitán (1995). Rodríguez Pose $(1997,1999)$ establece que las disparidades de la Unión Europea tienen una base geográfica y Paci (1997) señala la existencia de un proceso de convergencia en la productividad, lo que es compatible con la desigualdad en el ingreso por habitante. En el señalamiento de estructuras polarizadas destacan los trabajos de López Bazo et al. (1999) y el de Ezcurra (2001). 
numerosos estudios sobre la hipótesis de convergencia en nuestro país, ninguno de ellos incorpora variables ni métodos de análisis espacial, cuya pertinencia destacamos en la siguiente sección. ${ }^{10}$

\section{Convergencia espacial}

El análisis de convergencia del ingreso de acuerdo con numerosos autores europeos no puede realizarse sin considerar sus aspectos espaciales; Fingleton (1999), Rey y Montouri (1999) y Quah (1996) consideran que las regiones no pueden tratarse como si estuvieran aisladas, por lo cual debe considerarse que su crecimiento está vinculado con el de las áreas espacialmente contiguas.

Cheshire y Carbonaro $(1995,1996)$ cuestionan el enfoque tradicional de convergencia beta por no considerar los factores espaciales que influyen en el crecimiento regional. Badinger, Müller y Tonel (2001) realizan un estudio de convergencia espacial con un análisis de ecuaciones econométricas y datos de panel dinámico, considerando cortes transversales y series de tiempo. Sus conclusiones señalan que de no tomarse en cuenta estos factores espaciales, los modelos econométricos de convergencia presentan un problema de especificación incorrecta.

La metodología para incorporar efectos espaciales en los modelos econométricos ha sido desarrollada por la econometría espacial en los trabajos de Anselin (1988), Anselin y Florax (1995), Anselin y Bera (1998), Kelejian y Prucha (1998). En términos generales, proponen la estimación de ecuaciones econométricas que incorporan en la variable dependiente y en el término de error el efecto espacial asociado, sobre todo a la proximidad espacial.

No obstante, a pesar de los avances de este enfoque, se considera limitado, ya que la concepción de espacio sólo se refiere a la localización caracterizada por vecindad geográfica, lo cual implica estimar sólo la ubicación y proximidad de las áreas geográficas como significativas en el crecimiento, dejando de lado las interacciones

10 Esquivel (1999), Esquivel y Messmacher (2002) y Gamboa y Messmacher (2002) han documentado la evolución de las convergencias beta y sigma en el producto per capita estatal mexicano a partir de 1960. Destacan también otros trabajos sobre la hipótesis de convergencia por sus novedosas aplicaciones técnicas y métodos de evaluación, éste es el caso de Calderón, Cuauhtémoc y Tykonenko (2007) — que aplican un modelo de panel y un método de estimación bayessiano - y el de Rodríguez Oreggia, Eduardo (2007) — relevante por su análisis dinámico y por aplicar un modelo probabilístico. Sin embargo, a pesar del avance técnico y metodológico, su enfoque es sectorial y no dan ninguna importancia al espacio en su explicación. 
económicas entre áreas geográficas no contiguas. Su consideración requiere que se conciba el espacio como dimensión, como lo señala Asuad (2006) ${ }^{11}$ lo cual implica analíticamente incluir el efecto de las interacciones económicas en el espacio geográfico.

De ahí que al considerar el espacio como dimensión, se toman en cuenta dichas interacciones económicas espaciales, las cuales analíticamente se estiman como una relación funcional entre el crecimiento del ingreso y los niveles de concentración económica espacial. De tal manera que se considera que el análisis de convergencia espacial en el ingreso entre áreas geográficas, para valorar los efectos espaciales, debe incluir además de la proximidad espacial el análisis de sus efectos espaciales provocado por sus interacciones económicas en dicho espacio.

\section{Convergencia y concentración económica espacial}

La ecuación de la hipótesis de convergencia condicional, en la que se asumen diferentes estados estacionarios, fue estimada originalmente por Barro y Sala-i-Martin, utilizando mínimos cuadrados ordinarios (MCO) con datos de corte transversal y contrastando la hipótesis mediante la aplicación de una $t$ de significancia estadística al coeficiente $\beta$ de la ecuación. ${ }^{12}$ De tal manera que si la hipótesis nula $(b=0)$ era rechazada a favor de la opción ( $<<0$ ), se concluía que todas las regiones convergían al mismo nivel de ingreso per capita.

Este procedimiento de estimación fue cuestionado porque la ecuación estimada presentaba sesgo al omitir los niveles iniciales de tecnología, por no ser observables en la especificación econométrica. Islam (1995) propuso resolver el problema de

11 De acuerdo con este autor, la concepción de la dimensión espacial de la economía implica estimar la actividad económica integrada en el espacio geográfico. Es decir, se considera que los atributos espaciales de la actividad económica no se pueden separar de la actividad económica y se expresan en el espacio geográfico mediante el tamaño, localización, forma físico funcional, dirección y movilidad de la economía en el espacio geográfico. Por tanto, el análisis de la dimensión lleva implícito analizar sitios económicos, es decir, las áreas en el espacio geográfico donde se lleva a cabo la actividad económica y que se caracterizan por sus diferentes atributos espaciales y por las interacciones económicas que se realizan entre ellas.

12 La formulación econométrica de la ecuación de convergencia, de acuerdo con Sala $\sim$ i $\sim$ Martin (2002), es: $\log \left(y_{i, t}\right) \sim \log \left(y_{i, t-1}\right)=a \sim \beta \log \left(y_{i, t-1}\right)+u_{i t}$, en el cual la diferencia $\log \left(y_{i, t}\right) \sim \log \left(y_{i, t-1}\right)$ expresa la tasa de crecimiento del producto per capita entre el año $t \sim 1$ y el año $t, u_{i t}$ es un término de perturbación y $\beta$ es una constante positiva con un rango tal que $0<\beta<1$. Un mayor coeficiente $\beta$ implica mayor tendencia a la convergencia, a esta última se le conoce como convergencia beta o $\beta$-convergencia. La expresión condicionada de la ecuación implica la incorporación de variables adicionales que actúen como proxy del estado estacionario. 
especificación y estimación utilizando modelos de panel, en los cuales los efectos fijos permitían considerar los niveles iniciales de tecnología. Estudios recientes (Yudong y Weeks, 2000; Deininger y Olinto, 2000; Bond et al. 2001) han aplicado modelos de panel dinámico y lo que puede señalarse es que los diferentes procedimientos empleados para la estimación no han mostrado contundentemente que uno sea superior a otro. ${ }^{13}$

Un segundo cuestionamiento a los métodos de especificación y estimación de la ecuación de convergencia fue formulado por Quah (1996), al señalar que las regiones no podían ser evaluadas de forma aislada unas de otras. El crecimiento de una región se encuentra vinculado con el de otras, ello significa que en el término de perturbación aleatoria uit de la ecuación de la existencia de la hipótesis de convergencia condicional es posible que se presente un patrón de correlación o dependencia espacial. Ignorar esta situación da lugar a un problema de especificación en el modelo econométrico, por ello los análisis de convergencia han tendido a formular modelos espaciales y es justamente en esta perspectiva en la que se ubica el modelo que presentamos en las secciones siguientes. ${ }^{14}$ En este modelo se establece la importancia de incorporar variables y métodos de análisis espacial en la validación de la hipótesis de convergencia, destacando la concentración económica espacial como variable significativa y los métodos de análisis de la econometría espacial.

El enfoque teórico de la concentración económica espacial (CEE) bajo el enfoque de la dimensión espacial de la economía se sustenta en la propuesta teórica y metodológica de Asuad (2006), en la que se concibe a la CEE como la densidad económica que presenta la actividad económica sobre la unidad espacial que ocupa en relación con resto de unidades espaciales que le rodea. Su medición se realiza mediante un índice de participación, teniendo como variables representativas el valor agregado o la población ocupada.

No obstante, previamente a la aplicación del modelo, se requiere delimitar con precisión las unidades de análisis, en este caso, entidades federativas que se consideran regiones agrícolas, lo cual requiere un proceso de selección y delimitación.

13 El resultado de aplicar diferentes métodos de estimación afecta significativamente la velocidad de convergencia entre regiones de acuerdo con la metodología empleada.

14 En Arbia (2006) se revisan las técnicas más avanzadas en la estimación de modelos de convergencia espacial, en ellas se muestran modelos espaciales de panel, espaciales de Markov, espacio-temporales, de variables discretas, de externalidades espaciales, bayesianos espaciales y modelos no paramétricos. 


\section{Regionalización agrícola ${ }^{15}$}

A pesar de que el enfoque analítico de convergencia se ha aplicado mayoritariamente a la convergencia del crecimiento del ingreso total entre entidades federativas, existen varios análisis que se aplican a sectores específicos como la educación y la innovación tecnológica (Esquivel y Messmacher, 2002). Sin embargo, desde nuestro punto de vista en este caso se requiere previamente identificar y delimitar las regiones agrícolas en el país, tomando como base las entidades federativas, con el propósito de establecer con claridad las que se caracterizan propiamente como tales, a fin de aplicar el análisis de convergencia espacial que proponemos.

La regionalización agrícola que se elabora toma como criterios básicos la participación en la producción y en el empleo agrícola por entidad federativa, debido a la elevada asociación estadística que existe entre esas variables. Para delimitar esas regiones se elaboró un índice compuesto ponderado, que denominamos índice de regionalización agrícola (IRA), el cual recoge los efectos de ambas participaciones, esto es, del PIB agrícola y el nivel de empleo, medidos también por dos índices previos, como puede verificarse en el Anexo 1.

Los ponderadores para el cálculo del IRA se obtuvieron por medio de MCO, cuyo resultado arroja una contribución de 0.46 para el empleo y de 0.54 para el PIB. Los resultados de la regionalización agrícola permiten identificar 23 entidades federativas del país como agrícolas, las cuales, por su importancia, se dividen en cuatro regiones de acuerdo con el nivel de participación en la producción y en el empleo, como se presenta en el cuadro 1 y el mapa 1.

\section{Análisis de convergencia tradicional y espacial}

El análisis de la convergencia del ingreso entre las regiones agrícolas del país, considerando las diversas entidades que las integran, se realiza con la medición de la hipótesis de convergencia desde el enfoque tradicional y espacial. En el tradicional se validan las hipótesis de convergencia sigma ( $\sigma$-convergencia) y convergencia

15 Cabe aclarar que no obstante las limitaciones de la disponibilidad de información agrícola, se toman como variables aproximadas los datos agropecuarios del PIB y población ocupada (PO), validando estadísticamente su representatitividad. La metodología y el procedimiento de la regionalización se presenta en el Anexo1. 


\section{Cuadro 1}

Regiones agrícolas 1980-2004

\begin{tabular}{|c|c|c|}
\hline Regiones agrícolas & Valor índice & Entidades federativas \\
\hline Participación muy alta & 127 & Jalisco, Veracruz, Sinaloa, Sonora, Michoacán \\
\hline Participación alta & 106 & $\begin{array}{l}\text { Chiapas, Chihuahua, Guanajuato, México, Puebla, } \\
\text { Tamaulipas, Durango, Oaxaca }\end{array}$ \\
\hline Participación baja & 86 & $\begin{array}{l}\text { San Luis Potosí, Coahuila, Baja California, Nayarit, } \\
\text { Morelos, Zacatecas, Guerrero, Nuevo León }\end{array}$ \\
\hline Participación muy baja & 65 & Yucatán, Baja California Sur \\
\hline
\end{tabular}

Fuente: elaboración propia con base en información del anexo 1.

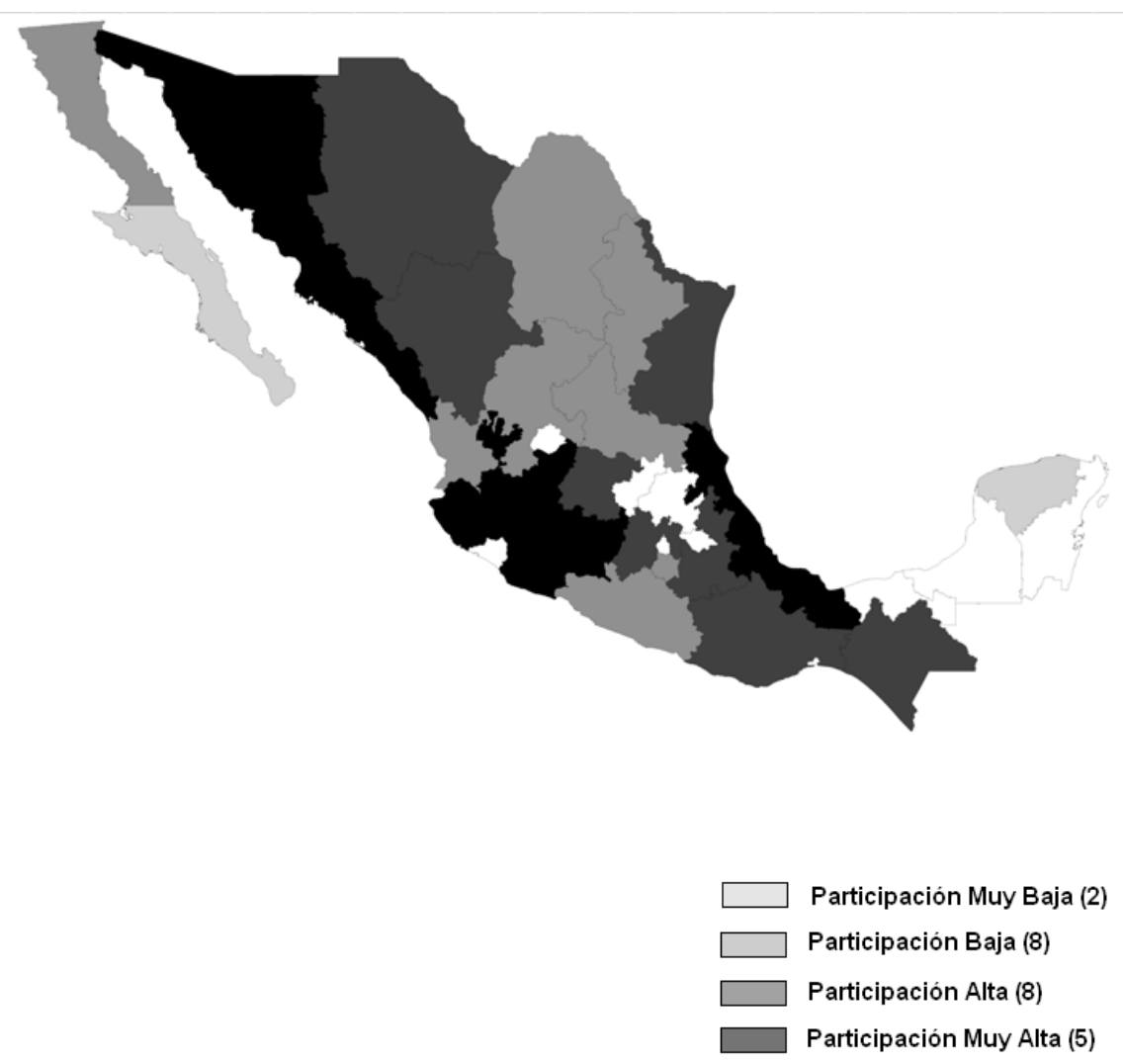


beta ( $\beta$-convergencia), mientras que en el espacial se incluyen los efectos de proximidad espacial y los de interacción económica espacial. ${ }^{16}$

\section{$\sigma$ convergencia}

El análisis de $\sigma$-convergencia se aplica al considerar como unidades de análisis tanto a las entidades federativas como a las regiones agrícolas, porque es fundamental distinguir las diferencias en la tendencia a la convergencia en el ingreso del conjunto de entidades federativas como integradas, constituyendo regiones por su semejanza estadística. Ello debido a que la convergencia o divergencia entre regiones puede mostrar la tendencia a la formación regional de núcleos de convergencia y divergencia del ingreso de las regiones agrícolas del país.

La hipótesis de convergencia tradicional en el largo plazo pretende determinar la evolución de la varianza del PIB per capita agrícola de las entidades federativas caracterizadas como agrícolas del país en el periodo 1970-2003; de acuerdo con el comportamiento de los datos, la tendencia de la dispersión del PIв per capita agrícola se muestra en la gráfica 1.

En la gráfica se observa que la tendencia de largo plazo en el sector agrícola del país ha sido a la divergencia, es decir, a la existencia de mayor disparidad del ingreso entre las entidades federativas del país, dado que mientras en 1970 la desigualdad del PIB agrícola per capita entre entidades agrícolas era de 0.33 , para 2003 alcanzó un valor de 0.44 . Sin embargo, en el corto plazo destacan dos periodos de convergencia en los cuales las entidades federativas agrícolas más pobres disminuyeron sus diferencias de ingreso con respecto de las más ricas: 1970 a 1977 y 1994 a 1999, coincidiendo este último con el inicio del tLCAN.

Por su parte, el comportamiento en la convergencia en el ingreso de las regiones agrícolas en el país, en el mismo periodo de análisis, muestra una evolución diferenciada. Por un lado, si bien se presentan procesos de convergencia regional del ingreso per capita, éstos se caracterizan por darse al interior de las regiones más ricas y más pobres, identificadas como regiones agrícolas de muy alta y muy baja participación,

${ }_{16}$ Existen dos conceptos usuales de convergencia, $\beta$-convergencia (a la que ya se aludió en la nota 12) y convergencia sigma o o-convergencia, la cual ocurre cuando la dispersión del ingreso per capita entre grupos de economías tiende a reducirse en el tiempo. En Sala i Martin (2002) se demuestra que la convergencia beta es una condición necesaria, aunque no suficiente para la existencia de la convergencia sigma. 


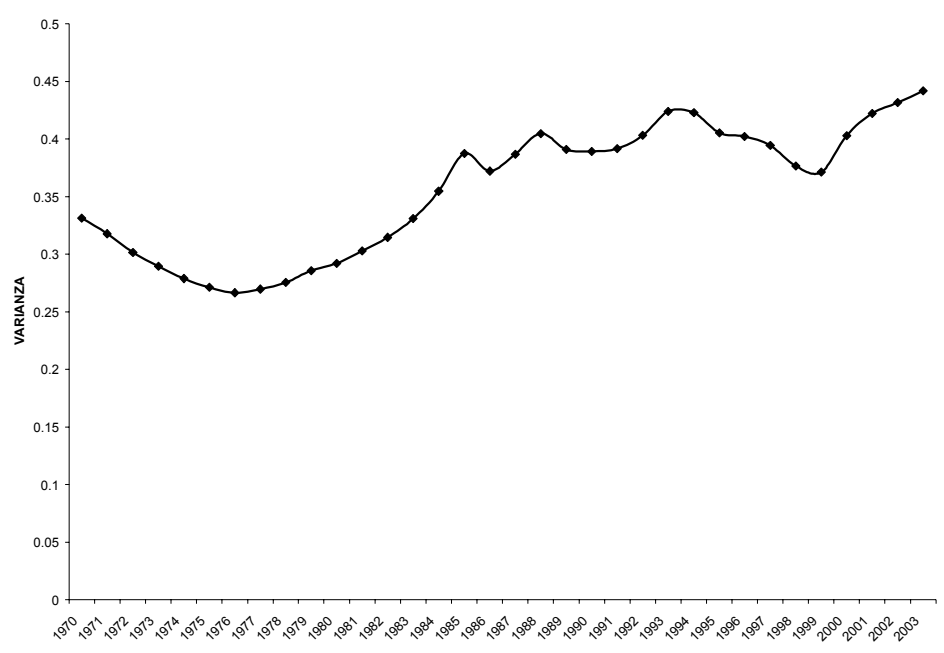

Gráfica 1

G-convergencia: varianza del PIB per capita de los estados del país, 1970-2003.

respectivamente. Lo cual implica, de hecho, una formación de núcleos de convergencia, en los cuales las entidades de las regiones más ricas y las que integran las más pobres tienden a converger entre sí, tal y como se observa en la gráfica 2.

De acuerdo con su comportamiento en el largo plazo, las regiones agrícolas de muy alta participación (MA) y las de muy baja participación (MB) tienden a disminuir la heterogeneidad en el ingreso per capita. La varianza de las regiones MA se redujo de 0.22 a 0.19 entre 1970 y 2003. Un patrón similar ocurre al interior de los estados que integran la región de menor participación agrícola MB, o más pobre; su varianza se redujo prácticamente a la mitad al pasar de 0.87 a 0.41 en el mismo periodo.

Estos resultados contrastan con la mayoría de las entidades federativas que conforman las regiones agrícolas de alta y baja participación, dado que la divergencia se incrementó en el largo plazo, al pasar las diferencias en sus ingresos per capita respectivamente de 0.30 a 0.50 y de 0.20 a 0.40 .

\section{$\beta$-convergencia}

Los resultados de la estimación de la hipótesis de $\beta$-convergencia para las entidades federativas agrícolas del país indican que no se puede validar estadísticamente la existencia de un proceso de largo plazo que haya llevado a disminuir los diferencia- 


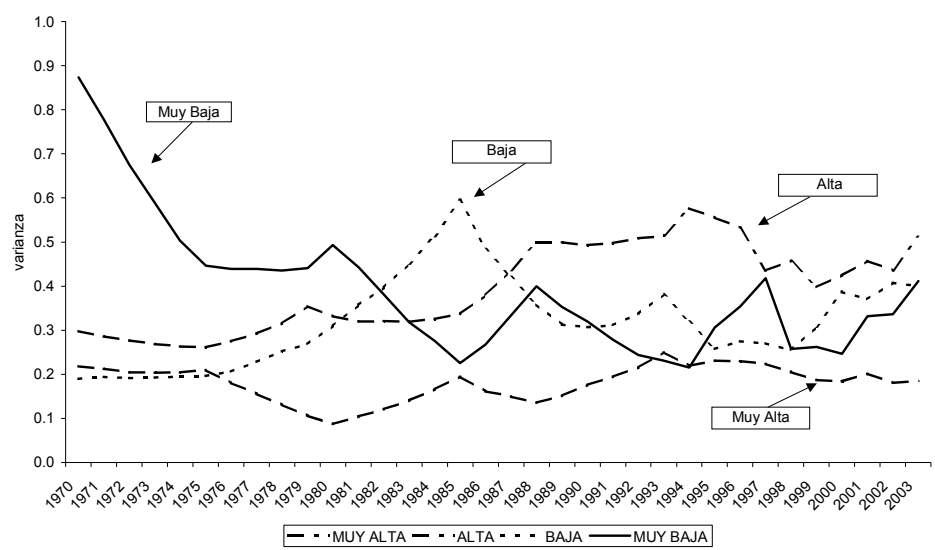

Gráfica 2

o-convergencia: varianza del PIB per capita entre las regiones agrícolas, 1970-2003.

les en el crecimiento del ingreso de esas regiones del país. El coeficiente $\beta$ no es estadísticamente significativo, con lo que se rechaza la hipótesis de convergencia. Por lo cual no se valida que las regiones de menor producción agrícola estén creciendo a tasas mayores que las de mayor producción (véase Anexo 2, cuadro 1).

\section{Convergencia espacial}

La validación del incumplimiento de la hipótesis de convergencia tradicional de las regiones agrícolas y la ratificación de la divergencia y el incremento de las disparidades en la producción y el ingreso entre entidades y regiones agrícolas requieren que se analice si en su comportamiento tienen incidencia los aspectos espaciales, es decir, la proximidad y las interacciones espaciales.

Este análisis se realiza mediante dos opciones analíticas; la primera consiste en dar evidencia empírica sobre las repercusiones que tienen los efectos espaciales en el modelo de convergencia tradicional, por lo que en la estimación econométrica de la ecuación de convergencia se incorpora la dependencia espacial en la variable dependiente y en el término de error, de acuerdo con los trabajos de Anselin (1988), Anselin y Florax (1995), Anselin y Bera (1998), Kelejian y Prucha (1998), por lo que acorde con esta propuesta, el modelo econométrico para la ecuación de convergencia se plantea como: 


$$
\begin{gathered}
\log \left(\frac{y_{i t}}{y_{i, t-T}}\right)=a-\rho W_{1} \log \left(\frac{y_{i t}}{y_{i, t-T}}\right)+\left(1-e^{-\beta T}\right) \log \left(y_{i, t-T}\right)+u_{i t, t-T} \\
u_{i t, t-T}=\lambda W_{2} u_{i t, t-T}+\mu
\end{gathered}
$$

En el cual el término de error $\mathrm{u}_{\mathrm{it}}$ incorpora una estructura de dependencia espacial autorregresiva, siendo $\mathrm{W}_{1}$ y $\mathrm{W}_{2}$ matrices de pesos espaciales. La noción de espacio es incorporada en las matrices de pesos Wi, las cuales se construyen con las proximidades espaciales entre las regiones. ${ }^{17}$

La segunda opción consiste en explicar los aspectos espaciales como una interacción económica espacial resultado de la relación funcional entre regiones, la cual depende de la asociación entre crecimiento del ingreso y los niveles de concentración económica espacial en las entidades federativas del país. Ésta se realiza de acuerdo con la propuesta de Asuad (2006), lo cual implica que en vez de pensar en los efectos espaciales asociados a la proximidad o vecindad geográfica de las unidades de producción, se considere su interacción. En ese sentido, el espacio dentro de la ecuación de convergencia se expresa como un factor funcional espacial asociado a la concentración económica espacial de los sitios económicos, de ahí que la ecuación de convergencia se modifique, incorporando una variable exógena que incorpora los efectos funcionales de la concentración económica en el espacio en relación con el ingreso per capita, como medida de la interacción, lo cual se denota como:

$$
\log \left(\frac{y_{i t}}{y_{i, t-T}}\right)=a+\left(1-e^{-\beta T}\right) \log \left(y_{i, t-T}\right)+\gamma X_{i, t-T}+u_{i t, t-T}
$$

En la cual $\mathrm{X}_{\mathrm{i}, \mathrm{t}}$ es una variable exógena que considera los efectos funcionales de la concentración económica en el espacio con respecto del ingreso.

17 Las proximidades se calculan utilizando distancias binarias con los vecinos y pueden ser de tipo torre, alfil o reina, véase al respecto Anselin (1988). 
Convergencia espacial dada por la proximidad geográfica

La estimación de la ecuación (1) corrobora la inexistencia de los efectos espaciales provenientes de la proximidad espacial en la convergencia beta, dado que se confirma que la velocidad de la convergencia entre las entidades federativas y regiones agrícolas del país no se ve afectada por la dependencia de la proximidad espacial para el periodo 1970-2003, ya que las pruebas de pesos espaciales de la ecuación (1) indican que no son estadísticamente significativos (Anexo 2, cuadro 2).

Este comportamiento se ratifica incluso por periodos, ya que no existe asociación entre la velocidad de convergencia y la dependencia espacial para los periodos 1985-2003 y 1994-2003, que corresponden respectivamente a las etapas de apertura de la economía mexicana y de la firma del tLCAN (véase Anexo 2, cuadros 3 al 10).

Para confirmar si la tasa de crecimiento del PIB per capita de las entidades federativas agrícolas se encuentra asociada regionalmente, se calculó el índice de Moran ${ }^{18}$ que es el indicador más usual para medir asociación espacial. Dicho índice se aplicó a la tasa de crecimiento del PIB per capita agrícola para todo el periodo 1970-2003, el resultado indica que la correlación espacial en las tasas de crecimiento es negativa y muy pequeña, -0.047 . Además de ser poco significativa, ya que el p-valor para la hipótesis nula de no correlación espacial fue 0.1620 (utilizando 999 permutaciones), es decir, no hay un efecto derrame en el crecimiento de una región a otra. ${ }^{19}$

18 El índice de Moran (1948) se define en la expresión siguiente;

$$
I=\frac{N}{S_{0}} \frac{\sum_{i j}^{N} w_{i j}\left(x_{i}-x\right)\left(x_{j}-x\right)}{\sum_{i=1}^{N}\left(x_{i}-x\right)^{2}}
$$

donde $\mathrm{x}_{\mathrm{i}}$ es la variable cuantitativa $\mathrm{x}$ en la región $\mathrm{i}, \overline{\mathrm{x}}$ es su media muestral, $\mathrm{w}_{\mathrm{ij}}$ son los pesos de la matriz de proximidad W, $\mathrm{N}$ es el tamaño de muestra y

$$
S_{0}=\sum_{i} \sum_{j} w_{i j}
$$

El índice de Moran sigue una distribución normal estandarizada en muestras grandes, de forma tal que un valor positivo (negativo) significativo del índice Z(I) llevará al rechazo de la hipótesis nula de no autocorrelación espacial y a la aceptación de autocorrelación espacial positiva (negativa).

19 Para el cálculo del índice de Moran se utilizó el paquete GeoDa desarrollado por Luc Anselin, disponible libremente en la dirección de internet: www.geoda.uiuc.edu 
Estos resultados sugieren que la tasa de crecimiento del PIB per capita agrícola no se encuentra clusterizada o aglomerada en regiones específicas, es decir, que estados con alto/bajo crecimiento no se encuentran asociados a estados con alto/ bajo crecimiento, sino que existe un patrón aleatorio en el ritmo de crecimiento del sector.

\section{Convergencia espacial y concentración económica espacial}

El análisis de la hipótesis de convergencia tradicional y el de efectos espaciales, dados por la proximidad geográfica de las entidades federativas, muestra la inexistencia de la convergencia y la tendencia a la divergencia en las regiones agrícolas del país. De ahí que se establezca la necesidad de probar la asociación estadística entre la divergencia regional y la concentración económica de la producción agrícola, a fin de validar la relación funcional que se establece entre el crecimiento del producto agrícola regional y el nivel de la concentración económica de la producción agrícola. En este comportamiento se asume que se da una relación directa entre mayor crecimiento de la producción y el nivel de concentración de la producción agrícola, por lo cual se establece una relación inversa entre la divergencia y la concentración económica de la producción. De tal forma que los resultados esperados de este análisis serían que la divergencia regional debería ser explicada por el nivel de concentración económica de la producción agrícola en las regiones.

Esta medición se realiza a partir de la evaluación del modelo de convergencia condicional asociada a la concentración económica espacial, la cual se establece como una relación funcional entre el producto regional agrícola y el nivel de su concentración económica espacial. Con base en ello se reformuló el modelo espacial de la ecuación (1), incorporando como variable aproximada, tanto las diferencias en estado estacionario como la de los efectos espaciales, a una variable que da cuenta de las diferencias de concentración económica entre los estados del país, que corresponde a la tasa de crecimiento de la concentración económica espacial, que se denota como $\log (\mathrm{Ct} / \mathrm{Ct}-\mathrm{i})$, correspondiente al logaritmo de la tasa de crecimiento en la concentración económica de la producción agrícola regional.

Los resultados de la estimación del modelo (2) en el largo plazo, de 1970 a 2003, confirman la inexistencia de la convergencia y, por tanto, validan la divergencia, destacando la concentración económica como variable significativa en su explicación, dado que tiene un efecto de $6.79 \%$ en el ritmo de crecimiento (Anexo 3 , cuadro 1). 
En los periodos 1970-1985 y 1985-2003 se confirma nuevamente la inexistencia de convergencia y también el efecto de la concentración es significativo. Aunque se observa que dicho efecto tiende a descender ligeramente (Anexo 3, cuadros 2 y 3).

Este comportamiento se vuelve a validar y se refuerza más aún, si se toma como umbral 1994 con la entrada en vigor del TLCAN. En el primer subperiodo 19701994, previo al TLCAN, se mantuvo la divergencia entre los estados del país, siendo el efecto de la concentración significativo y con un coeficiente menor al de largo plazo. Pero en el segundo periodo, 1994-2003, posterior al TLCAN, el efecto de la concentración económica espacial es significativo y superior a su valor de largo plazo, además de que el coeficiente beta es positivo y significativo, dando cuenta con ello de que en el proceso de divergencia de ese periodo la concentración económica de la producción es determinante del comportamiento divergente de la producción agrícola de las regiones del país (Anexo 3, cuadros 4 y 5).

\section{Impacto de la politica nacional y cambio estructural}

El impacto de la política nacional hacia la divergencia regional a partir de la apertura de la economía mexicana podría dar lugar a un cambio estructural positivo si existiese una asociación entre el comportamiento del ingreso del sector agrícola por entidad federativa y región y el cambio en la estructura productiva hacia el empleo industrial y los servicios, ya que se validaría que se está dando un desplazamiento de la población de sector de baja productividad a los de alta, lo cual también implica una asociación positiva con el aumento de la densidad de población y con la población urbana. Por el contrario, si los efectos son negativos, se tendría un crecimiento del empleo del sector primario y un aumento de la dispersión poblacional, lo cual se reflejaría en el aumento de las localidades no urbanas y dispersas, es decir, con menor de 15000 habitantes.

De tal forma que para analizar los efectos en la divergencia regional sobre el cambio estructural, se formuló el siguiente modelo.

$$
\ln _{\mathrm{y}}=x_{1}-x_{2} \ln P i-x_{3} \ln P i i-x_{4} \ln P i i i-x_{5} \ln D u-x_{6} \ln G u-x_{7} \ln \operatorname{Pr}-e
$$

En el cual:

$\mathrm{Y}=$ variable dependiente, es el PIB per capita agrícola

$\mathrm{Pi}=$ empleo población ocupada sector primario. Participación

Pii = empleo población ocupada sector secundario. Participación

Piii = empleo población ocupada sector terciario. Participación 
$\mathrm{Du}=$ densidad de población: habitantes $/ \mathrm{km}$

$\mathrm{Gu}=$ grado de urbanización: participación población urbana sobre el total

$\mathrm{CPr}=$ población rural menor de 15000 habitantes. Participación en la población total

La evaluación del modelo primero se aplicó al total de las entidades federativas del país, para el periodo 1990-2000, y sus resultados muestran que no existe asociación entre el ingreso del sector agrícola de dichas entidades y el cambio en la estructura productiva hacia la industria y los servicios, no obstante que se presenta asociación positiva con significancia estadística respecto de la densidad de población, con un efecto negativo en la productividad agrícola, lo cual se interpreta como desplazamiento de la población rural hacia las zonas urbanas, sin afectar positivamente el producto y el empleo secundario y terciario; ello indicaría desempleo y probablemente aumento del empleo informal y terciarización improductiva de la economía. Condición que reflejaría los impactos negativos de la política de apertura en el desarrollo económico y social de esas regiones (Anexo 4, cuadro 1).

No obstante, el cambio estructural por regiones presenta cambios significativos entre las regiones de mayor y menor participación agrícola en el periodo 19902000. Para este análisis se agruparon las regiones en dos grupos: las de alta —muy alta y alta — y baja participación — muy baja y baja—, a fin de analizar los efectos en el cambio estructural de la divergencia regional.

Los resultados para la región de mayor participación muestran que no hay evidencia de cambio estructural favorable, las variables no fueron significativas con excepción de la densidad urbana que tiene un efecto negativo en la productividad agrícola, lo que implica aumentos en el desplazamiento de población rural a la urbana y probablemente aumento de sector informal (Anexo 4, cuadro 2).

Por su parte, en la región de baja participación los resultados muestran significancia estadística para la participación del empleo en el sector primario, lo cual afecta negativamente la productividad del sector y de manera positiva el número de localidades rurales, aumentándolas a la vez que crece el grado de urbanización; el que se traduce en una ruralización empobrecida y una terciarización improductiva de los centros urbanos (Anexo 4, cuadro 3). 


\section{Conclusiones}

1. Se confirma la hipótesis de la tendencia a largo de plazo de la divergencia en el ingreso y en la velocidad de crecimiento de las regiones agrícolas del país en el periodo de estudio.

2. Se identifican reducidos periodos de tendencia a la convergencia de las diferencias del ingreso per capita de las entidades federativas agrícolas del país de 1970 a 1977 y de 1994 a 2003

3. Se establece la tendencia a la convergencia regional del ingreso entre las entidades de las regiones más ricas y las correspondientes a las más pobres. De esta manera, Jalisco, Veracruz, Sinaloa, Sonora y Michoacán, que integran la región más rica, tienden a acercarse entre sí. Lo mismo sucede con Yucatán y Baja California Sur, que constituyen la región agrícola más pobre del país, lo que implica la formación de núcleos de convergencia de ingreso entre las entidades agrícolas más ricas y las más pobres.

4. La mayoría de las entidades agrícolas del territorio se caracteriza por una tendencia al aumento de la desigualdad en su ingreso. Entre las regiones agrícolas de mayor participación están Chiapas, Chihuahua, Guanajuato, México, Puebla, Tamaulipas, Durango y Oaxaca. Mientras que las que integran la región agrícola de baja participación son San Luis Potosí, Coahuila, Baja California, Nayarit, Morelos, Zacatecas, Guerrero y Nuevo León.

5. Se confirma la inexistencia de los efectos espaciales provenientes de la proximidad espacial en la convergencia sigma y en la beta, dado que la velocidad de la convergencia entre las entidades federativas y regiones agrícolas del país no se ve afectada por la dependencia de la proximidad espacial para el periodo 1970-2003.

6. Se valida la inexistencia de la asociación entre la velocidad de convergencia y la dependencia espacial para los periodos 1985-2003 y 1994-2003, que corresponden a las etapas de apertura de la economía mexicana y de la firma del TLCAN, por lo cual no se identifica una influencia de la proximidad espacial en la convergencia para estos periodos.

7. Se validan la divergencia en el ingreso y su crecimiento de las regiones agrícolas en el largo plazo de 1970 a 2003, destacando la concentración económica como variable significativa en su explicación, pues tiene un efecto de $6.79 \%$ en el ritmo de crecimiento. 8. En los periodos 1970-1985 y 1985-2003 se confirma nuevamente la inexistencia de convergencia y el efecto de la concentración es significativo. Aunque se observa que este efecto tiende a descender ligeramente. 
9. El comportamiento de divergencia y el papel fundamental de la concentración económica espacial se vuelve a confirmar y se refuerza aún más, si se toma como año de umbral a 1994 con la entrada en vigor del TLCAN. En el primer subperiodo 1970-1994, previo al TLCAN, se mantuvo la divergencia entre los estados del país, siendo el efecto de la concentración significativo y con un coeficiente menor al de largo plazo. Pero en el segundo periodo 1994-2003, posterior al TLCAN, el efecto de la concentración económica espacial es significativo y superior a su valor de largo plazo, además de que el coeficiente beta es positivo y significativo, dando cuenta con ello de que en el proceso de divergencia de ese periodo la concentración económica de la producción es determinante del comportamiento divergente de la producción agrícola de las regiones.

10. Los resultados de análisis del cambio estructural de 1990-2000 muestran que no existe asociación entre el ingreso del sector agrícola de las entidades federativas del país y el cambio en la estructura productiva hacia la industria y los servicios. No obstante, se presenta asociación positiva con significancia estadística respecto de la densidad de población, con un efecto negativo en la productividad agrícola, lo cual se interpreta como un desplazamiento de la población hacia las zonas urbanas, sin repecutir positivamente en el empleo secundario y terciario, lo que parece indicar desempleo y probablemente aumento del empleo informal.

11. Los resultados para la región de mayor participación muestran que no hay evidencia de cambio estructural, las variables no fueron significativas con excepción de la densidad urbana que tiene un efecto negativo en la productividad agrícola, lo cual se interpreta como aumentos en el desplazamiento de población rural a la urbana, reduciendo la producción agrícola sin tener efecto en el producto y en el empleo industrial y servicios productivos y sí en un crecimiento urbano improductivo. 12. Por su parte, en la región de baja participación, los resultados muestran significancia estadística para la participación del empleo en el sector primario, lo que afecta de manera negativa la productividad del sector y positivamente el número de localidades rurales al incrementarlas, a la vez que aumenta el grado de urbanización, lo que se traduce en una ruralización empobrecida y una terciarización improductiva de los centros urbanos.

13. De ahí que se considere que la apertura de la economía mexicana y el TLCAN han propiciado divergencia regional en las entidades que conforman las zonas agrícolas, donde la concentración económica en las regiones de mayor participación refuerzan las disparidades. Por otra parte, sus efectos en el cambio estructural son 
nulos y, por el contrario, agudizan los problemas estructurales de la economía mexicana y del sector agrícola, al reforzar los patrones de ruralización empobrecida y de terciarización urbana improductiva, elevando la ineficiencia de ambos e incrementando costos e ineficiencia productiva que redunda en descensos en los niveles de vida y bienestar de los agricultores y trabajadores del sector rural en general. De ahí la necesidad de reformular la política en la cual el empleo, el ingreso y la eficiencia permitan resolver los problemas estructurales sectoriales y espaciales del sector agrícola, así como reducir los niveles de pobreza y aumentar el bienestar de la población mediante políticas deliberadas regionales y territoriales de producción y empleo en el sector agrícola.

\section{Bibliografía}

Álvarez Ayuso, Inmaculada y María de Jesús Delgado Rodríguez, "Difusión tecnológica y convergencia económica: un análisis de las regiones y los sectores productivos españoles" en Economía en Sociedad y Territorio, vol. v, núm. 18, 2005, pp. 301-322.

Arbia, Giuseppe; "Spatial Econometrics, Statistical Foundations and Applications to Regional Convergence", en Springer, Berlín, 2006.

Asuad Sanén, Normand Eduardo, Un ensayo teórico y metodológico sobre el proceso de concentración económica espacial y su evidencia empírica en la región económica megalopolitana de 1970 a 2000 y sus antecedentes, México, UnAM, 2006, en preparación para su publicación.

Asuad Sanén, Normand Eduardo, Luis Quintana Romero y Roberto Ramírez Hernández, "Desarrollo y políticas regionales en México: Retos y perspectivas 2006-2020", en José Luis Calva Téllez (Coordinador), Políticas de Desarrollo Regional, vol. 13, UNAM, Miguel Ángel Porrúa, Cámara de Diputados, 2007, pp. 231-264.

, Luis Quintana Romero y Roberto Ramírez Hernández, "Desarrollo y políticas urbanas en México: Retos y perspectivas 2006-2020”, en José Luis Calva Téllez(Coordinador), Políticas de desarrollo regional, vol. 13, unAM, Miguel Ángel Porrúa 2007, Cámara de Diputados, 2007. pp. 353-383.

- Economía regional y urbana: Introducción a las teorías, técnicas y metodologías básicas, Puebla, Benemérita Universidad Autónoma de Puebla y Colegio de Puebla, A. C, 2001, pp. 1-403.

Anselin, Luc, Spatial econometrics: Methods and models, Dordrecht, Kluwer Academic Publishers, 1988

y A. Bera, "Spatial dependence in linear regression models with an introduction to spatial econometrics", en D. Giles y A. Ullah (editores), Handbook of applied economic statistics, New York, Marcel Dekker, 1998, pp. 237-289.

- y J. Florax, "New directions in spatial econometrics: Introduction", en: L. Anselin y J. Florax (editores), New directions in spatial econometrics, Berlin, Heidelberg, New York, Springer, 1995.

Badinger, Harald y G. Müller Werner y Gabriele Tondl, "Regional Convergence in the European Union (1985-1999): A Spatial Dynamic Panel Analysis" en HWWA Discussion, Hamburg, núm. 210, Institute of International Economics, 2001.

Barro, R. y Xavier Sala-i-Martin, "Economic Growth and Convergence across the United States”, NBER Working, núm. 3419, 1990. , "Convergence across States and Regions", en Brooking Papers on Economic Activity, núm. 1, 1991, pp. 107-182.

- "Convergence", en Journal of Political Economy, núm. 100, 1992, pp. 407-443.

, Economic Growth, New York, McGrawHill, 1995.

Boldrin, M. y F. Canova, "Inequality and Convergence in Europe's regions; reconsidering 
European regional policies", en Economic Policy, núm. 32, 2001, pp. 207-245.

Bond, S. y A. Hoeffler y J. Temple, "GMm estimation of empirical growth models", en CEPR discussion, núm. 3048, Centre for Economic Policy Research, Londres, 2001.

Calderón, Cuauhtémoc y A. Tykonenko, "Convergencia regional e inversión extranjera directa en México en el contexto del TLCAN, 1994-2002”, en Investigación Económica, vol. LXVI, núm. 259, Facultad de Economía, UNAM, enero-marzo de 2007, pp.15-41.

Canova, F., "Testing for Convergence clubs in income per capita: a predictive density approach" en International Economic Review, núm. 45, 2004, pp.49-77.

_ , y A. Marcel, "The poor stay poor: non convergences across countries and regions", en CERP working, núm. 1405, 1995.

Cheshire, P. y G. Carbonaro, "ConvergenceDivergence in Regional Growth Rates: An Empty Black Box?", en H. Armstrong, R. Vickerman (editores), Convergence and Divergence among European Regions, Pion, Londres, 1995.

, "Urban economic growth in Europe: testing theory and policy prescriptions", en Urban Studies, núm. 33, 1996, pp. 1111-1128.

Cass, D., "Optimum Growth in anAggregative Model of Capital Accumulation", en Review of Economic Studies, vol. xxxII, 1965,pp. 223-240.

Deininger, K. yP.Olinto, "Asset distribution, inequality and growth", en World Bank working paper, núm. 2375, The World Bank, Washington, 2000.

Dewhurst, J. y H. Mutis-Gaitan, "Varying speeds of regional GDP p.c. convergence in the European Union 1981-1991", en H. Armstrong y R.W. Viekerman (editores), Convergence and divergence among the European Regions, Pion, Londres, 1995, pp. 40-45.

Diamond, P., "National Debt in a Neoclassical Growth Model", American Economic Review, núm. 55, 1965, pp. 1126-1150.

Dunford, M., "Regional Disparities in the European Community: Evidence from the REGIO Databank", Regional Studies, núm. 27, 1993, pp. 727-743.

Esquivel, G y M. Messmacher, "Sources of regional non convergence in Mexico", Working paper. The World Bank, 2002.

_, Daniel Lederman, Miguel Messmacher y Renata Villoro, Why NAFTA Did Not Reach the South, junio de 2002.
Esteban, J., "La desigualdad interregional en Europa y en España: Descripción y análisis", en Crecimiento y convergencia regional en España y Europa, vol. 2, Instituto de Análisis Económico-CSIC y Fundación de Economía Analítica, Barcelona, 1994, pp. 13-82.

Ezcurra, Roberto, Convergencia y cambio estructural en la Unión Europea, Departamento de Economía, Universidad Pública de Navarra, 2001.

Fingleton, F., "Estimates of Time to Economic Convergence: An Analysis of Regions of the European Union", en International Regional Science, núm 22, 1999, pp. 5-34.

Fingleton, B., R. Lewney y D. Pinelli, "Regional Growth and Convergence", en EC's Single Market Review, vol. 1, subserie vi, Kogan Page, Londes 1996.

Grossman, G., y E. Helpman, Innovation and Growth in the Global Economy, MIT Press, Cambridge, 1991.

, "Endogenous Innovation in the Theory of Growth", en Journal of Economic Perspectives, núm. 8, 1994, pp. 23-44.

Hernández Laos, Enrique, "La desigualdad regional en México", en Rolando Cordera Campos y Carlos Tello (coordinadores), $L a$ desigualdad en México, México, Siglo XXI, 1984, p. 595.

Islam, N., "Growth empirics: A panel data approach", en Quarterly Journal of Economics, núm. 110(4), 1995, pp. 1127-1170.

Kelejian, H. y I. Prucha, “A generalized spatial two-stage least squares procedure for estimating a spatial autoregressive model with autoregressive disturbances", en Journal of Real Estate Finance and Economics, 1998, pp. 99-121.

Koopmans, T., "On the Concept of Optimal Ecomomic Growth", The Econometric Approach to Development Planning, North Holland, Amsterdam, 1965.

Léonard E. y B. Losch, "L'insertion de l'agriculture mexicaine dans le marché nordaméricain: changements structurels, mutations de l'action publique et recompositions de l'économie rurale et régionale", en Colloque RINOS-CEIM, UQaM, Intégrations régionales et stratégies de développement, Montréal, junio de 2005, 27 pp.

López Bazo, E., E. Vayá, A. Mora y J. Surinach, "Regional Economic Dynamics and Convergence in the European Union", en 
The Annals of Regional Science, vol.33, núm. 3, 1999, pp.343-370.

Lucas, R, "On the Mechanics of Economic Development", en Journal of Monetary Economics, 22 , núm.1, 1988, pp. 3-42.

Mankiw, G., P. Romer y D. Weil, "A contribution to the empirics of economic growth", en Quarterly Journal of Economics, vol. cVII, núm. 2, 1992, pp. 407-437.

Mendoza, Miguel Angel, Capital Humano y Crecimiento Regional, Tesis unAm, 2005.

Messmacher, Miguel, Alfredo Cuevas y Alejandro Werner, Changes in the Patterns of External Financing in Mexico, since the approval of NAFTA, Banco de México, Julio de 2002.

Moran, P.A.P., "The interpretation of statistical maps", en Journal of the Royal Statistical Society, B-10, 1948, pp.243-251.

Neven, D. y C Gouyette, "Regional Convergence in the European Community", en Journal of Common Market Studies núm. 33, 1995, pp. 47-65.

Paci, R. y F. Pigliaru, "Structural change and convergence: an Italian regional perspective", en Structural Change and Economic Dynamics, num. 8, 1997, pp. 297-318.

Quah, D., "Regional Convergence Cluster across Europe", en European Economic Review núm. 40, 1996, pp. 951-958.

Rello, F., El campo en la encrucijada nacional, Foro 2000, Secretaría de Educación Pública, México, 1986.

comportamiento agrícola y en el desarrollo rural, Banco Mundial, 2006.

Rey, Sergio J. y Brett, D. Montouri, "U.S. Regional Income Convergence: A Spatial Econometric Perspective", en Regional Studies, núm. 33, 1999, pp.143-156.

Rodríguez Oreggia, Eduardo, "Winners and Losers of Regional Growth in México and Their dynamics", en Investigación Económica, Facultad de Economía, UNAM, vol. LXVI, 259, enero-marzo de 2007, pp.43-62.

, "El papel del factor estatal en la percepción de la convergencia regional en la Unión Europea", en Información comercial española, núm. 762, 1997, pp. 9-24.

Rodriguez Pose, A., "Convergence or Divergence? Types of Regional Responses to SocioEconomic Change in Western Europe", en Tijdschrift voor Economische en Sociale Geografie, 90, núm. 4, 1999, pp. 363-378.
Romer, P., "Increasing returns and long-run growth", en Journal of Political Economy, vol. 94, núm. 5, 1986, pp. 1002-1037.

Romer, P., "Growth Based on Increasing Returns due to Specialization", en American Economic Review Papers and Proceedings, vol. 77, núm. 2, 1987, pp. 56-62.

Romer, P., "Crazy Explanations for the Productivity Slowdown", en NBER Macroeconomics Annual, núm. 2, 1987, pp. 163-210.

Sala-i-Martin, Xavier, On Growth and States, tesis doctoral, Harvard University, 1990.

- Apuntes de crecimiento económico, Antoni Bosch, Barcelona, 1994a.

, "La riqueza de las naciones. Evidencia y teoría sobre crecimiento regional y convergencia”, en Moneda y Crédito, núm. 198, 1994b, pp. 13-55.

, "Regional Cohesion: Evidence and Theories of Regional Growth and Convergence", en European Economic Review, núm. 40, 1996, pp. 1325-1352.

Solow, R., "A Contribution to the Theory of Economic Growth", en Quarterly Journal of Economics, vol. LXx, núm. 1, 1956, pp. 65-94.

Soto, Germán, "Generación del producto interno bruto mexicano por entidad federativa, 19401992", Trimestre Económico, núm. 287, Fondo de Cultura Económica, 2005, pp. 617654.

Terrasi, M., "National and Spatial Factors in EU Regional Convergence", en J. R. Cuadrado-Roura, M. Parellada (coordinadores), Regional Convergence in the European Union, Springer-Verlag, Berlín, 2002.

USTR, "NAFTA at Seven" en Seven Year report http:/ /www.ustr.gov/regions/whemisphere/ nafta.shtml, Washington, DC; 2001.

Yudong y M. Weeks, "Provincial income convergence in China, 1953-1997: A panel data approach", DAE working, paper 0010, University of Cambridge, Octubre, 2000.

Yúnez Naude, Antonio y Fernando Bárcenas Paredes, Lessons from NAFTA: The Case of Mexico's Agricultural Sector, World Bank, 2002. 


\section{Anexo 1}

\section{Regionalización}

Debido a la escasez de información agrícola, la identificación y delimitación de las regiones agrícolas del país presentó limitaciones para realizar los análisis que se pretenden. Baste recordar que el último censo agropecuario en nuestro país se llevó a cabo en 1991 y que el próximo se tiene planeado hasta 2007. Los datos disponibles no permiten identificar el producto interno agrícola por entidad federativa que es la información clave para el comportamiento del sector, por lo cual se utiliza como dato aproximado el PIB agropecuario. No obstante, se considera que la representación del comportamiento del sector agrícola mediante el comportamiento de la producción agropecuaria es adecuada, ya que se identificó la asociación entre la producción agropecuaria y el valor de la producción agrícola con un coeficiente de correlación de 0.95 , con la siguiente asociación gráfica.

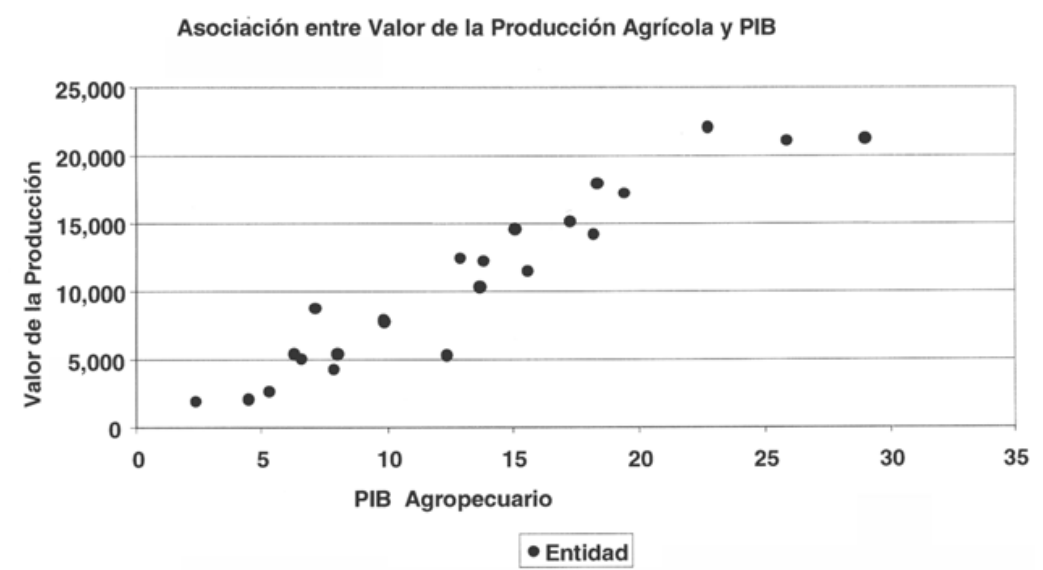

Además se identificó la asociación entre el PIB agropecuario y el empleo agrícola, al obtener un coeficiente de correlación de $\mathbf{0 . 7 5}$, con la siguiente asociación gráfica.

De ahí que se considere que el comportamiento de los datos del sector agropecuario es representativo del sector agrícola. Por otra parte, cabe señalar que se considera de manera preliminar como unidad de análisis a las entidades federativas del país, para su clasificación como regiones agrícolas, dado que la identificación 


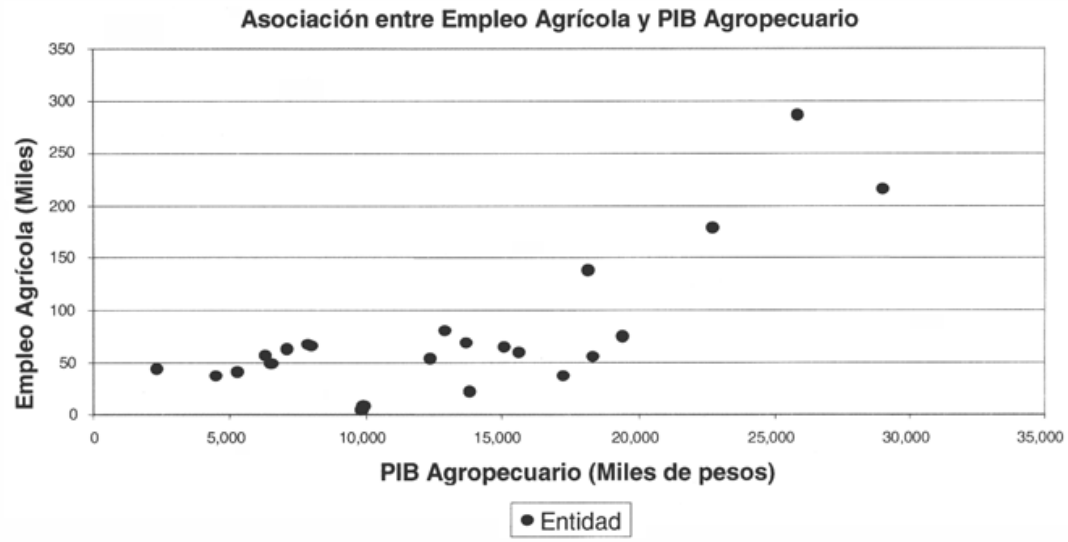

de áreas económicas funcionales agrícolas requiere de información detallada por municipio y por las áreas económicas básicas que contiene y de su validación en campo.

Se elaboraron dos índices simples, participación en la producción y en el empleo que posteriormente se ponderaron mediante un análisis de regresión. Los índices se elaboraron con una base de datos mediante información del INEGI (PIB agropecuario por año y población ocupada por entidad federativa). Además, se utilizaron las siguientes bases de datos:

1. SIACON de SAGARPA (datos de producción, valor de la producción, superficies sembrada y cosechada en toneladas por año y por entidad federativa).

2. Registros de trabajadores agropecuarios asegurados del IMSS (permanentes y eventuales) por entidad federativa.

En todos los casos la información abarca el periodo 1980-2004 aunque para efectos de estos cálculos, se emplearon únicamente los años 1980, 1985, 1995 y 2004.

El cálculo de los índices involucró la transformación de la escala de los datos de PIB y PO por medio de la fórmula siguiente:

$$
\begin{gathered}
\operatorname{In}\left(P I B_{i}\right)-\operatorname{In}(\max P I B) \\
\operatorname{In}(\max P I B-\operatorname{In}(\min P I B)
\end{gathered}
$$

Donde $P I B_{\mathrm{i}}$ es el $P I B / P O$ de la entidad i, mientras que max $P I B / \mathrm{PO}$ es el valor máximo del $P I B / P O$ en los años de estudio y $\min P I B / P O$ es el valor mínimo. 
Las regionalizaciones se realizaron mediante datos agrupados generados de tablas de frecuencia estadísticas, clasificando los estados de acuerdo con su índice observado sobre las clases creadas según la distribución de los datos.

Regionalización con datos agrupados para el índice de regionalización agrícola (IRA)

\begin{tabular}{|c|c|c|c|c|c|c|}
\hline \multirow[b]{2}{*}{ Jalisco } & $\begin{array}{l}\text { agrícola } \\
\text { acumulado }\end{array}$ & \multicolumn{2}{|l|}{$\begin{array}{l}\text { Empleo } \\
\text { Agrícola }\end{array}$} & \multicolumn{3}{|c|}{$\operatorname{IRA}$} \\
\hline & 1.000 & 0.930 & 1 & Jalisco & 1.968 & \\
\hline Veracruz & 0.955 & 1.000 & 2 & Veracruz & 1.955 & Pond (PIB) $=0.54$ \\
\hline Sinaloa & 0.903 & 0.883 & 3 & Sinaloa & 1.850 & $\operatorname{Pond}(E m p)=0.46$ \\
\hline Michoacán & 0.840 & 0.668 & 4 & Sonora & 1.732 & \\
\hline Chiapas & 0.818 & 0.596 & 5 & Michoacán & 1.687 & \\
\hline Sonora & 0.815 & 0.820 & 6 & Chiapas & 1.632 & \\
\hline México & 0.794 & 0.499 & 7 & Chihuahua & 1.575 & \\
\hline Chihuahua & 0.753 & 0.613 & 8 & Guanajuato & 1.572 & \\
\hline Guanajuato & 0.740 & 0.634 & 9 & México & 1.563 & \\
\hline Oaxaca & 0.705 & 0.361 & 10 & Puebla & 1.538 & \\
\hline Puebla & 0.701 & 0.646 & 11 & Tamaulipas & 1.534 & \\
\hline Tamaulipas & 0.678 & 0.687 & 12 & Durango & 1.471 & \\
\hline Durango & 0.661 & 0.586 & 13 & Oaxaca & 1.412 & \\
\hline Zacatecas & 0.572 & 0.101 & 14 & San Luis Potosí & 1.323 & \\
\hline Guerrero & 0.571 & 0.000 & 15 & Coahuila & 1.316 & \\
\hline San Luis Potosí & 0.489 & 0.639 & 16 & Baja California & 1.270 & \\
\hline Coahuila & 0.480 & 0.643 & 17 & Nayarit & 1.209 & \\
\hline Baja California & 0.442 & 0.626 & 18 & Morelos & 1.206 & \\
\hline Morelos & 0.408 & 0.562 & 19 & Zacatecas & 1.159 & \\
\hline Nayarit & 0.392 & 0.602 & 20 & Guerrero & 1.111 & \\
\hline Nuevo León & 0.324 & 0.520 & 21 & Nuevo León & 1.103 & \\
\hline Yucatán & 0.259 & 0.496 & 22 & Yucatán & 1.027 & \\
\hline \multirow[t]{2}{*}{ Baja California Sur } & 0.000 & 0.538 & 23 & Baja California Sur & 0.787 & \\
\hline & & & & Mediana = & 1.471 & \\
\hline
\end{tabular}

Regiones agrícolas 1980-2004

\begin{tabular}{|c|c|c|}
\hline Regiones agrícolas & Valor índice & Entidades federativas \\
\hline Participación muy alta & 127 & Jalisco, Veracruz, Sinaloa, Sonora, Michoacán \\
\hline Participación alta & 106 & $\begin{array}{l}\text { Chiapas, Chihuahua, Guanajuato, México, } \\
\text { Puebla, Tamaulipas, Durango, Oaxaca }\end{array}$ \\
\hline Participación baja & 86 & $\begin{array}{l}\text { San Luis Potosí, Coahuila, Baja California, Nayarit, } \\
\text { Morelos, Zacatecas, Guerrero, Nuevo León }\end{array}$ \\
\hline Participación muy baja & 65 & Yucatán, Baja California Sur \\
\hline
\end{tabular}


Anexo 2

Análisis de convergencia espacial

Cuadro 1

Convergencia $\beta$ en el sector agrícola, 1970-2003

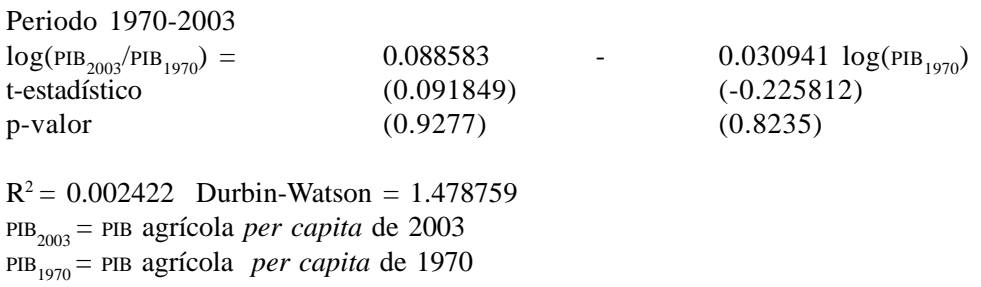

$\mathrm{PIB}_{1970}=$ PIB agrícola per capita de 1970

Cuadro 2

Diagnóstico de dependencia espacial, 1970-2003

Prueba

Índice de Moran (error)

$M I / D F$
0.045455
1
1
1
1
2

Valor

Multiplicadores de Lagrange (rezago)

LM Robusta (rezago)

Multiplicadores de Lagrange (error)

LM Robusta (error)

Multiplicadores de Lagrange (SARMA)

$0.5227273-0.4696806$

$0.0000000 \quad 0.9999997$

$0.5227273 \quad 0.4696806$

$0.0000000 \quad 0.9999996$

$0.5227273 \quad 0.7700009$

* Ninguna de las probabilidades es menor a los niveles de significancia usuales, por lo cual no se puede rechazar la hipótesis nula de no existencia de efectos espaciales.

Cuadro 3

Convergencia beta en el sector agrícola, 1970-1985

Periodo 1970-1985

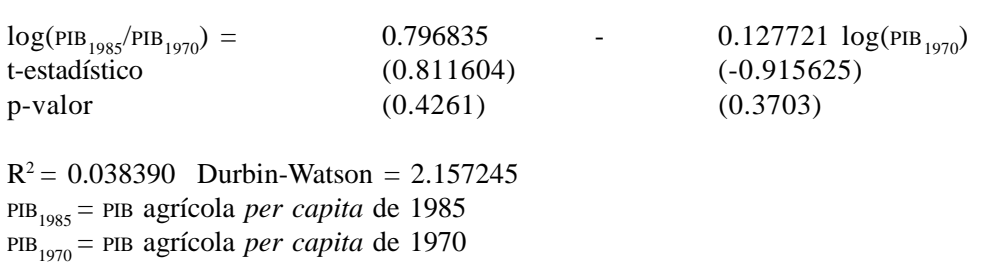


Cuadro 4

Diagnóstico de dependencia espacial, 1970-1985

\begin{tabular}{lccc}
\hline Prueba & MI/DF & Valor & PROB* \\
& & & $\mathrm{N} / \mathrm{A}$ \\
Índice de Moran (error) & 0.045455 & $\mathrm{~N} / \mathrm{A}$ & 0.4696806 \\
Multiplicadores de Lagrange (rezago) & 1 & 0.5227273 & 0.9999996 \\
LM robusta (rezago) & 1 & 0.0000000 & 0.4696806 \\
Multiplicadores de Lagrange (error) & 1 & 0.5227273 & 0.9999996 \\
LM robusta (error) & 1 & 0.0000000 & 0.7700009 \\
Multiplicadores de Lagrange (SARMA) & 2 & 0.5227273 & \\
\hline
\end{tabular}

* Ninguna de las probabilidades es menor a los niveles de significancia usuales, por lo cual no se puede rechazar la hipótesis nula de no existencia de efectos espaciales.

Cuadro 5

Convergencia $\beta$ en el sector agrícola, 1985-2003

\begin{tabular}{lll}
\hline $\log \left(\mathrm{PIB}_{2003} / \mathrm{PIB}_{1985}\right)=$ & $\begin{array}{l}0.958412 \\
(0.993542)\end{array}$ & $\begin{array}{l}0.142794 \log \left(\mathrm{PIB}_{1985}\right) \\
\text { t-estadístico }\end{array}$ \\
p-valor & & $(-1.027824)$ \\
\end{tabular}

$\mathrm{R}^{2}=0.047896$ Durbin-Watson $=1.600686$

$\mathrm{PIB}_{1985}=$ PIB agrícola per capita de 1985

$\mathrm{PIB}_{2003}=$ PIB agrícola per capita de 2003

\begin{tabular}{lccc}
\hline & \multicolumn{3}{c}{ Cuadro 6 } \\
Prueba & Diagnóstico de dependencia espacial 1985-2003 & \\
& $M I / D F$ & Valor & PROB* \\
\hline Índice de Moran (error) & 0.045455 & $\mathrm{~N} / \mathrm{A}$ & $\mathrm{N} / \mathrm{A}$ \\
Multiplicadores de Lagrange (rezago) & 1 & 0.5227273 & 0.4696806 \\
LM robusta (rezago) & 1 & -0.0000000 & -1.0000000 \\
Multiplicadores de Lagrange (error) & 1 & 0.5227273 & 0.4696806 \\
LM robusta (error) & 1 & -0.0000000 & -1.0000000 \\
Multiplicadores de Lagrange (SARMA) & 2 & 0.5227273 & 0.7700009 \\
\hline
\end{tabular}

* Ninguna de las probabilidades es menor a los niveles de significancia usuales, por lo cual no se puede rechazar la hipótesis nula de no existencia de efectos espaciales.

Cuadro 7

Convergencia $\beta$ en el sector agrícola, 1970-1994

\begin{tabular}{lcc}
\hline $\log \left(\mathrm{PIB}_{1994} / \mathrm{PIB}_{1970}\right)=$ & -0.107894 & $0.011883 \log \left(\mathrm{PIB}_{1970}\right)$ \\
$\mathrm{t}$-estadístico & $(-0.128279)$ & $(-0.099439)$ \\
$\mathrm{p}$-valor & $(0.8991)$ & $(0.9217)$ \\
& \\
$\mathrm{R}^{2}=0.000471 \quad$ Durbin-Watson $=1.778156$ & \\
$\mathrm{PIB}_{1970}=\mathrm{PIB}$ agrícola per capita de 1970 & \\
$\mathrm{PIB}_{1994}=\mathrm{PIB}$ agrícola per capita de 1994 & \\
\hline
\end{tabular}


Cuadro 8

Diagnóstico de dependencia espacial, 1970-1994

\begin{tabular}{lccc}
\hline Prueba & MI/DF & Valor & PROB* \\
& & & $\mathrm{N} / \mathrm{A}$ \\
Índice de Moran (error) & -0.045455 & $\mathrm{~N} / \mathrm{A}$ & 0.4696806 \\
Multiplicadores de Lagrange (rezago) & 1 & 0.5227273 & 0.9999999 \\
LM robusta (rezago) & 1 & 0.0000000 & 0.4696806 \\
Multiplicadores de Lagrange (error) & 1 & 0.5227273 & 0.9999999 \\
LM robusta (error) & 1 & 0.0000000 & 0.7700009 \\
Multiplicadores de Lagrange (SARMA) & 2 & 0.5227273 & \\
\hline
\end{tabular}

* Ninguna de las probabilidades es menor a los niveles de significancia usuales, por lo cual no se puede rechazar la hipótesis nula de no existencia de efectos espaciales.

Cuadro 9

Convergencia $\beta$ en el sector agrícola, 1994-2003

\begin{tabular}{lcc}
\hline $\log \left(\mathrm{PIB}_{2003} / \mathrm{PIB}_{1994}\right)=$ & 0.104152 & $0.006065 \log \left(\mathrm{PIB}_{1994}\right)$ \\
t-estadístico & $(-0.128279)$ & $(-0.099439)$ \\
$\mathrm{p}$-valor & $(0.7731)$ & $(0.9083)$ \\
& \\
$\mathrm{R}^{2}=0.000647$ Durbin-Watson $=$ & 1.783847 \\
$\mathrm{PIB}_{2003}=$ PIB agrícola per capita de 2003 & \\
$\mathrm{PIB}_{1994}=$ PIB agrícola per capita de 1994
\end{tabular}

Cuadro 10

Diagnóstico de dependencia espacial, 1994-2003

\begin{tabular}{lccc}
\hline Prueba & $M I / D F$ & Valor & PROB \\
& & & $\mathrm{N} / \mathrm{A}$ \\
Índice de Moran (error) & -0.045455 & $\mathrm{~N} / \mathrm{A}$ & 0.4696806 \\
Multiplicadores de Lagrange (rezago) & 1 & 0.5227273 & -1.0000000 \\
LM robusta (rezago) & 1 & -0.0000000 & 0.4696806 \\
Multiplicadores de Lagrange (error) & 1 & 0.5227273 & -1.0000000 \\
LM robusta (error) & 1 & -0.0000000 & 0.7700009 \\
Multiplicadores de Lagrange (sARMA) & 2 & 0.5227273 &
\end{tabular}

* Ninguna de las probabilidades es menor a los niveles de significancia usuales, por lo cual no se puede rechazar la hipótesis nula de no existencia de efectos espaciales. 
Anexo 3

Convergencia y concentración económica espacial

Cuadro 1

Convergencia $\beta$ y concentración económica espacial, 1970-2003

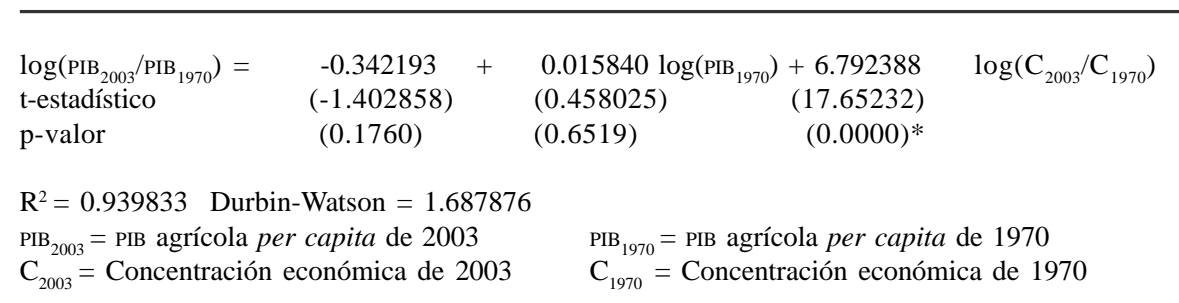

* Estadísticamente significativo, se rechaza la hipótesis nula de que el coeficiente de la concentración es cero.

Cuadro 2

Convergencia $\beta$ y concentración económica espacial, 1970-1985

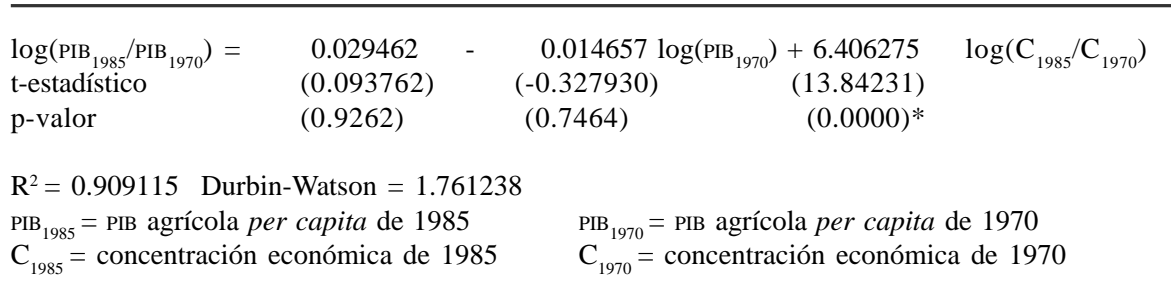

* Estadísticamente significativo, se rechaza la hipótesis nula de que el coeficiente de la concentración es cero.

Cuadro 3

Convergencia $\beta$ y concentración económica espacial, 1985-2003

$\begin{array}{lclc} & & & \\ \log (\mathrm{PIB} 2003 / \mathrm{PIB} 1985) & -0.370163 & 0.030571 \log \left(\mathrm{PIB}_{1985}\right)+6.764552 \log \left(\mathrm{C}_{2003} / \mathrm{C}_{1985}\right) \\ \text { t-estadístico } & (-2.859615) & (1.652295) & (35.40116) \\ \text { p-valor } & (0.0097) & (0.1141) & (0.0000)^{*}\end{array}$

$\mathrm{R}^{2}=0.985044$ Durbin-Watson $=2.490348$

$\mathrm{PIB}_{1985}=$ PIB agrícola per cápita de 1985

$\mathrm{C}_{1985}=$ concentración económica de $1985 \quad \mathrm{C}_{2003}=$ concentración económica de 2003

* Estadísticamente significativo, se rechaza la hipótesis nula de que el coeficiente de la concentración es cero. 
Cuadro 4

Convergencia $\beta$ y concentración económica espacial, 1970-1994

\begin{tabular}{|c|c|c|c|}
\hline $\begin{array}{l}\log \left(\mathrm{PIB}_{1994} / \mathrm{PIB}_{1970}\right)= \\
\text { t-estadístico } \\
\text { p-valor }\end{array}$ & $\begin{array}{c}-0.303557 \\
(-1.739106) \\
(0.0974)\end{array}$ & $\begin{array}{c}-0.006512 \\
(-0.262919) \\
(0.7953)\end{array}$ & $\begin{array}{c}\log \left(\mathrm{PIB}_{1970}\right)+6.534293 \log \left(\mathrm{C}_{1994} / \mathrm{C}_{1970}\right) \\
(21.65456) \\
(0.0000)^{*}\end{array}$ \\
\hline $\begin{array}{l}\mathrm{R}^{2}=0.959113 \text { Durb } \\
\mathrm{PIB}_{1970}=\text { PIB agrícola } p \\
\mathrm{C}_{1970}=\text { Concentración }\end{array}$ & $\begin{array}{l}\text { Watson }=1.6 \\
\text { capita de } 19 \\
\text { conómica de }\end{array}$ & $\begin{array}{l}\mathrm{PIB}_{1994} \\
\mathrm{C}_{1994}\end{array}$ & $\begin{array}{l}\text { IB agrícola per capita de } 1994 \\
\text { oncentración económica de } 1994\end{array}$ \\
\hline
\end{tabular}

* Estadísticamente significativo, se rechaza la hipótesis nula de que el coeficiente de la concentración es cero.

Cuadro 5

Convergencia y concentración económica espacial, 1994-2003

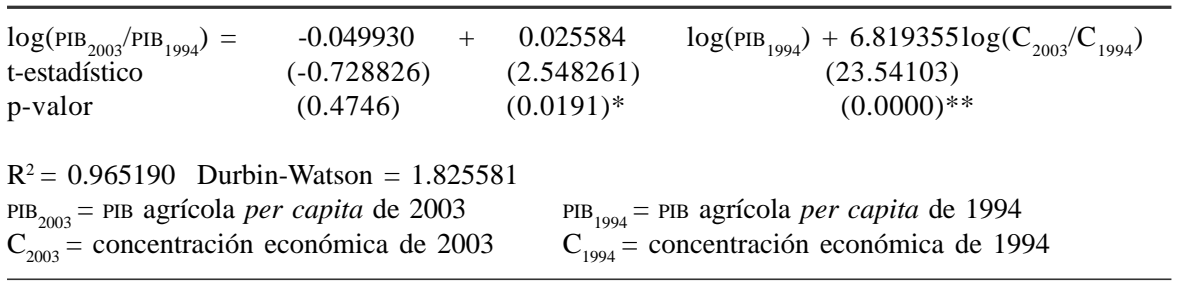

* Estadísticamente significativo, el signo positivo indica divergencia.

** Estadísticamente significativo, se rechaza la hipótesis nula de que el coeficiente de la concentración es cero.

\section{Anexo 4}

Cambio estructural

Cuadro 1

Ingreso y cambio estructural, 1990-2000

Modelo de Panel

Corte transversal: 23 observaciones

YI $=$

t-estadístico

p-valor

2156.272

$(3.905141)$

(0.0008)

Total de observaciones en el panel: 46

$\mathrm{R}^{2}=0.543887$ 
Cuadro 2

Ingreso y cambio estructural de las regiones de mayor participación, 1990-2000

Modelo de Panel

\begin{tabular}{lcc}
\hline Corte transversal: & 13 observaciones & Total de observaciones en el panel: 26 \\
& & \\
$\mathrm{YI}=$ & 2130.567 & $-10.05248 \mathrm{DU}$ \\
$\mathrm{t}$-estadístico & $(5.679551)$ & $(-2.571138)$ \\
$\mathrm{p}$-valor & $(0.0001)$ & $(0.0245)$ \\
& & \\
$\mathrm{R}^{2}=0.641759$ & & \\
\hline
\end{tabular}

Cuadro 3

Ingreso y cambio estructural de las regiones de menor participación: 1990-2000 Modelo de Panel

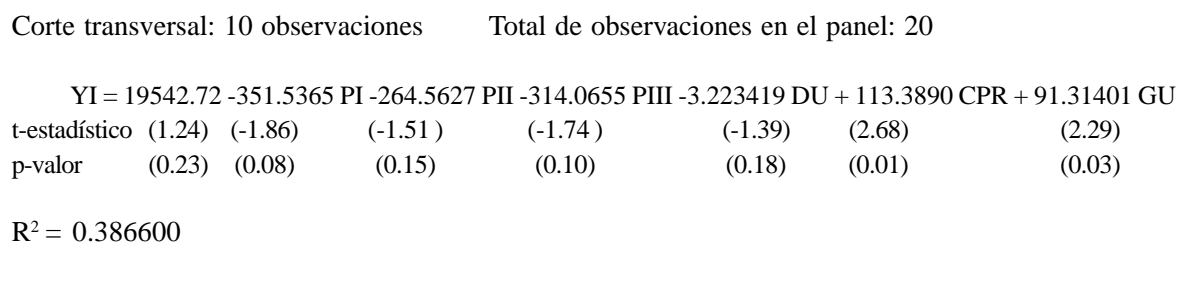




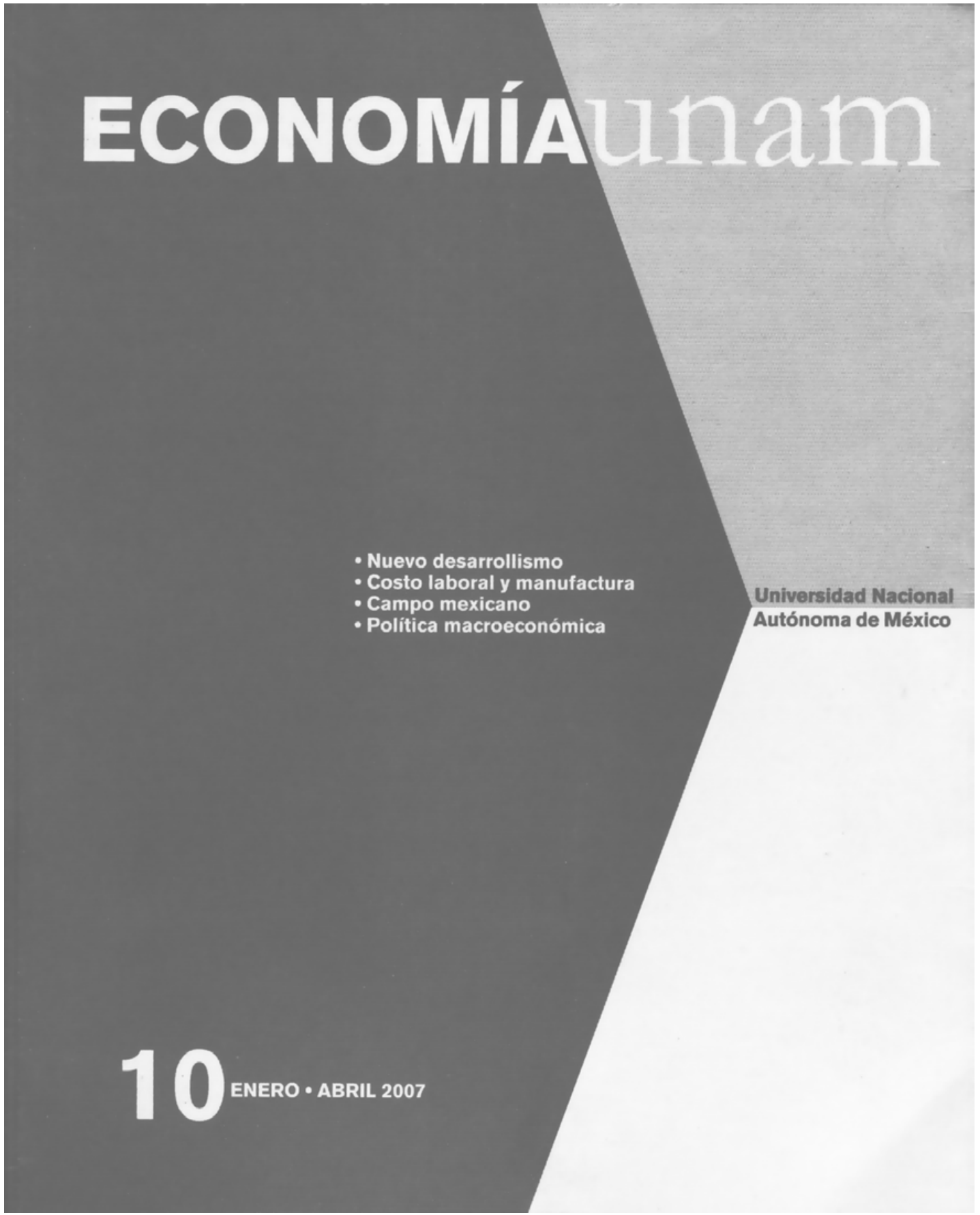

